\title{
Circulating tumour cell liquid biopsy in selecting therapy for recurrent cutaneous melanoma with locoregional pelvic metastases: a pilot study
}

Stefano Guadagni ${ }^{*}$, Giammaria Fiorentini ${ }^{2}$, loannis Papasotiriou ${ }^{3}$, Panagiotis Apostolou ${ }^{4}$, Francesco Masedu ${ }^{1}$, Donatella Sarti ${ }^{2}$, Antonietta Rossella Farina ${ }^{1}$, Andrew Reay Mackay ${ }^{1}$ and Marco Clementi $^{1}$

\begin{abstract}
Objectives: Circulating tumour cells (CTCS) from liquid biopsies provide an exceptional opportunity to obtain realtime tumour information and are under current investigation in several cancers, including cutaneous melanoma, but face significant drawbacks in terms of non-standardised methodology, low viable cell numbers and accuracy of CTC identification. In this pilot study, we report that chemosensitivity assays using liquid biopsy-derived metastatic melanoma (MM) CTCs, from 7 patients with stage IIIC, BRAF wild-type metastatic melanomas, localized exclusively to the pelvic region, un-eligible for immunotherapy and treated with melphalan hypoxic pelvic perfusion (HPP), is both feasible and useful in predicting response to therapy. Viable MM CTCs ( $>5$ cells $/ \mathrm{ml}$ for all 7 blood samples), enriched by transient culture, were characterised in flow cytometry-based Annexin V-PE assays for chemosensitivity to several drugs.
\end{abstract}

Results: Using melphalan as a standard, chemosensitivity cut-off values of $>60 \%$ cell death, were predictive of patient RECIST 1.1 response to melphalan HPP therapy, associated with calculated 100\% sensitivity, $66.67 \%$ specificity, $33.33 \%$ positive predictive, $100 \%$ negative predictive, and $71.43 \%$ accuracy values. We propose that the methodology in this study is both feasible and has potential value in predicting response to therapy, setting the stage for a larger study.

Trial registration Clinical Trials.gov Identifier NCT01920516; date of trial registration: August 6, 2013

Keywords: Liquid biopsies, Circulating tumour cells, Precision oncotherapy, Recurrent melanoma, Hypoxic pelvic perfusion, Melphalan, Pelvic loco-regional metastases

\section{Introduction}

Approximately $10 \%$ of cutaneous melanomas (CMs) recur as locoregional metastases following treatment [1]. Of these, $\approx 15 \%$ involve the pelvis [2], with only $2 \%$ of pelvic/inguinal metastases presenting in the absence of leg

\footnotetext{
*Correspondence: stefano.guadagni@univaq.it

1 Department of Applied Clinical Sciences and Biotechnology, University of L'Aquila, 67100 L'Aquila, Italy

Full list of author information is available at the end of the article
}

lesions [3]. Over the past 15 years, local, regional and systemic treatments for locoregional metastatic melanoma have evolved. For patients with stage III and IV CM with pelvic locoregional metastases, specialist melanoma centres provide local, regional, and systemic treatment options. Local treatments include surgical resection [4], topical therapy with diphencyprone or imiquimod [5], diathermy-fulguration, cryotherapy, laser ablation, radiofrequency ablation, intralesional injection with Rose Bengal [6] or Talimogene laherparepvec [7] or Darumon or

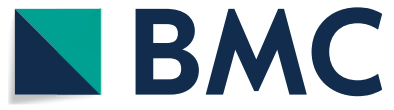

(c) The Author(s) 2020. This article is licensed under a Creative Commons Attribution 4.0 International License, which permits use, sharing, adaptation, distribution and reproduction in any medium or format, as long as you give appropriate credit to the original author(s) and the source, provide a link to the Creative Commons licence, and indicate if changes were made. The images or other third party material in this article are included in the article's Creative Commons licence, unless indicated otherwise in a credit line to the material. If material is not included in the article's Creative Commons licence and your intended use is not permitted by statutory regulation or exceeds the permitted use, you will need to obtain permission directly from the copyright holder. To view a copy of this licence, visit http://creativeco mmons.org/licenses/by/4.0/. The Creative Commons Public Domain Dedication waiver (http://creativecommons.org/publicdomain/ zero/1.0/) applies to the data made available in this article, unless otherwise stated in a credit line to the data. 
Coxsackie Virus A-21, and electro-chemotherapy $[8,9]$. Regional treatment options include regional radiation therapy $[10,11]$ and hypoxic pelvic perfusion (HPP), as isolated limb perfusion and isolated limb infusion are not anatomically practical for metastases targeting. HPP is achieved by blocking the blood circulation in the pelvic area at the aorta and inferior vena cava with balloon catheters and at thigh-level with pneumatic cuffs. The HPP rational, as for isolated limb infusion, is based upon the potential to expose locoregional melanoma metastases to higher drug concentrations, with the cytotoxicity of agents, such as melphalan, augmented by hypoxia [12]. We recently reported that melphalan HPP in 36 pretreated patients with stage III and IV melanoma, with pelvic locoregional metastases in progression, resulted in an overall response rate of $94 \%$, a median survival time of 15 months and a 5 -years survival rate of $8 \%$ [3, 13-15]. Systemic therapies, either alone or in combination with local and/or regional therapies, are currently proposed when pelvic melanoma metastases are considered unresectable for technical and clinical reasons. In patients with unresectable stage III and IV, dabrafenib and trametinib $B R A F / M E K$ inhibitors have resulted in 3 -year overall survival (OS) rates of 44\%, pembrolizumab, a monoclonal anti-PD-1 antibody, has resulted in 4-year OS rates of $44 \%$ and the combination of ipilimumab anti-CLTA-4 and nivolumab anti-PD-1 antibodies has resulted in three-year OS rates of $58 \%$. However, in these studies, published over the past 5 years, unresectable stage III melanomas represent only $3 \%$ of cases, making the extrapolation of these data to patients with unresectable stage III melanoma localized to the pelvic region, challenging [16]. Furthermore, target therapy only provides a significant improvement in overall median survival for $\approx 50 \%$ of $\mathrm{BRAF}^{\mathrm{V} 600 \mathrm{E}}$ mutated patients $[17,18]$ and new immunotherapies are effective in $\approx 45 \%$ of $B R A F$ wild-type patients [19-21]. Finally, patients with concomitant autoimmune disorders, chronic viral infections, organ dysfunction, organ transplant, brain metastases, or too old and frail, or pregnant, were in general excluded from immune checkpoint inhibitor clinical trials [22].

This apparent plethora of therapeutic options not only reflects the fact that not all treatments are available in each institution but also that no single strategy is suitable for every patient, with treatment choice dependent upon lesion number, size, anatomic location, the presence of regional lymph node or distant metastases and also biomolecular aspects, concomitant disease and previous therapy. For these reasons, we believe that treatment strategies for pelvic locoregional metastases should be multidisciplinary and could benefit greatly from the detailed characterisation of biomolecular characteristics and relative chemosensitivity of metastatic melanoma cells, a possibility that is offered by liquid biopsies, using purified circulating tumour cells (CTCs) obtained from individual patients. This method has been approved for prognosis by USA Food and Drug Administration [23] and despite a lack of methodological consensus, is under investigation as a potential method for identifying therapeutic strategies for cancers, including melanoma [24-26].

Here, we report a pilot study of CTCs purified from a homogeneous group of stage IIIC melanoma patients with locoregional, $B R A F$ wild-type metastases located exclusively to pelvic region, who were not eligible for immune checkpoint inhibitor therapy and were submitted for melphalan HPP therapy. The aim of this study was to confirm both the feasibility and utility of assessing the chemosensitivity of CTCs purified from liquid biopsies, as a predictive test for selecting therapeutic strategy.

\section{Main text}

This project was performed in accordance with the Declaration of Helsinki and was approved by the ethics committee of ASL n.1, Abruzzo, Italy (10/CE/2018, 19 July, n.1419). Written informed consent was obtained from each patient. From a prospective trial of melanoma patients undergoing melphalan perfusion/hypoxic infusion (Clinical Trials. gov Identifier NCT01920516), a subset of 41 were selected with stage III and IV patients with locoregional metastases located in the pelvis and/ or inguinal region and/or upper third of the thighs. From this 41-patient cohort, 7 patients were selected with $B R A F$ wild-type status, stage IIIC, not eligible for immunotherapy, submitted to melphalan HPP (Table 1A). Histopathological analysis revealed that all metastases exhibited an epithelioid phenotype. BRAF status and $M G M T$ promoter methylation status were assessed as previously described [14]. Exclusion from immunotherapy was due to concomitant Hepatitis $\mathrm{C}$ infection in 4 patients, in treatment with sofosbuvir $(400 \mathrm{mg})$ and daclatasvir $(30 \mathrm{mg})$ and acute phase inflammatory bowel disease in 3 patients, treated with high dose corticosteroids. This study adheres to CONSORT guidelines.

CTC purification and chemosensitivity assays are detailed in Additional file 1 and have been described previously $[27,28]$. Briefly, metastatic melanoma CTCs were purified from blood samples by column-based magnetic cell separation, using CD45 magnetic beads. Purified CTCs were qRT-PCR validated for CD63 expression and assayed for sensitivity to chemotherapeutic agents in Annexin V-PE flow cytometry chemosensitivity assays. Surgical and percutaneous HPPs, followed by chemofiltration (Fig. 1a), were performed as previously described [13, 29]. Briefly, for HPP, pelvic circulation was isolated by blocking aortic and inferior vena cava blood flow with 


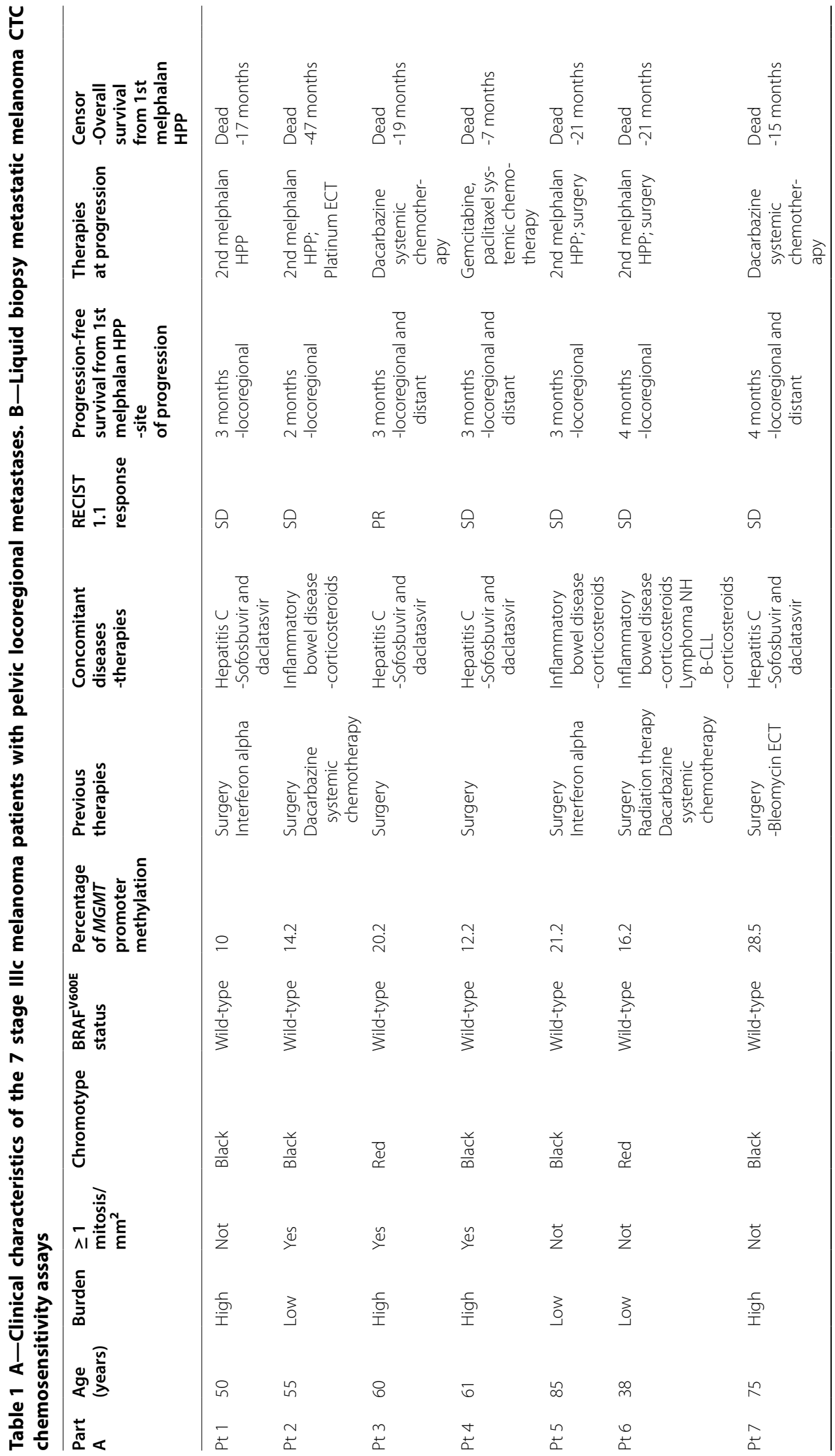




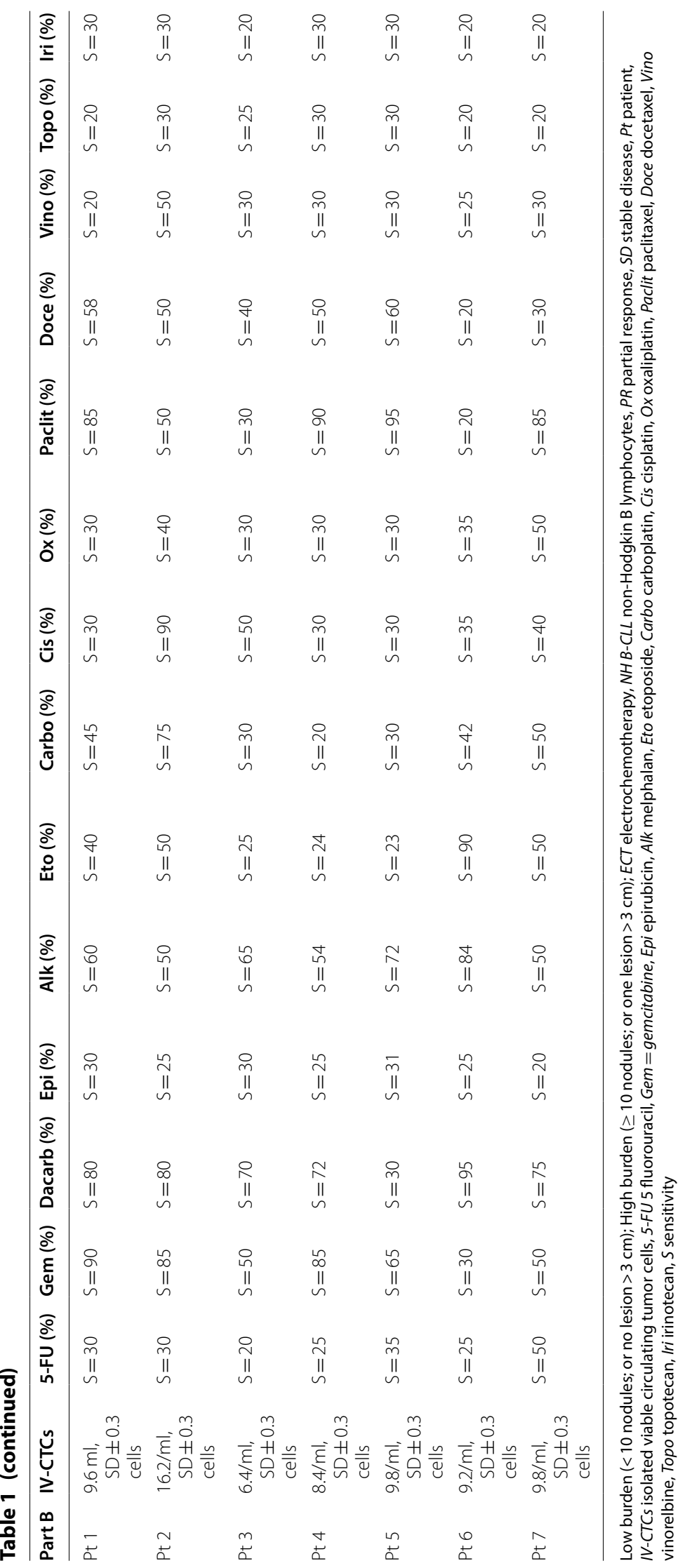




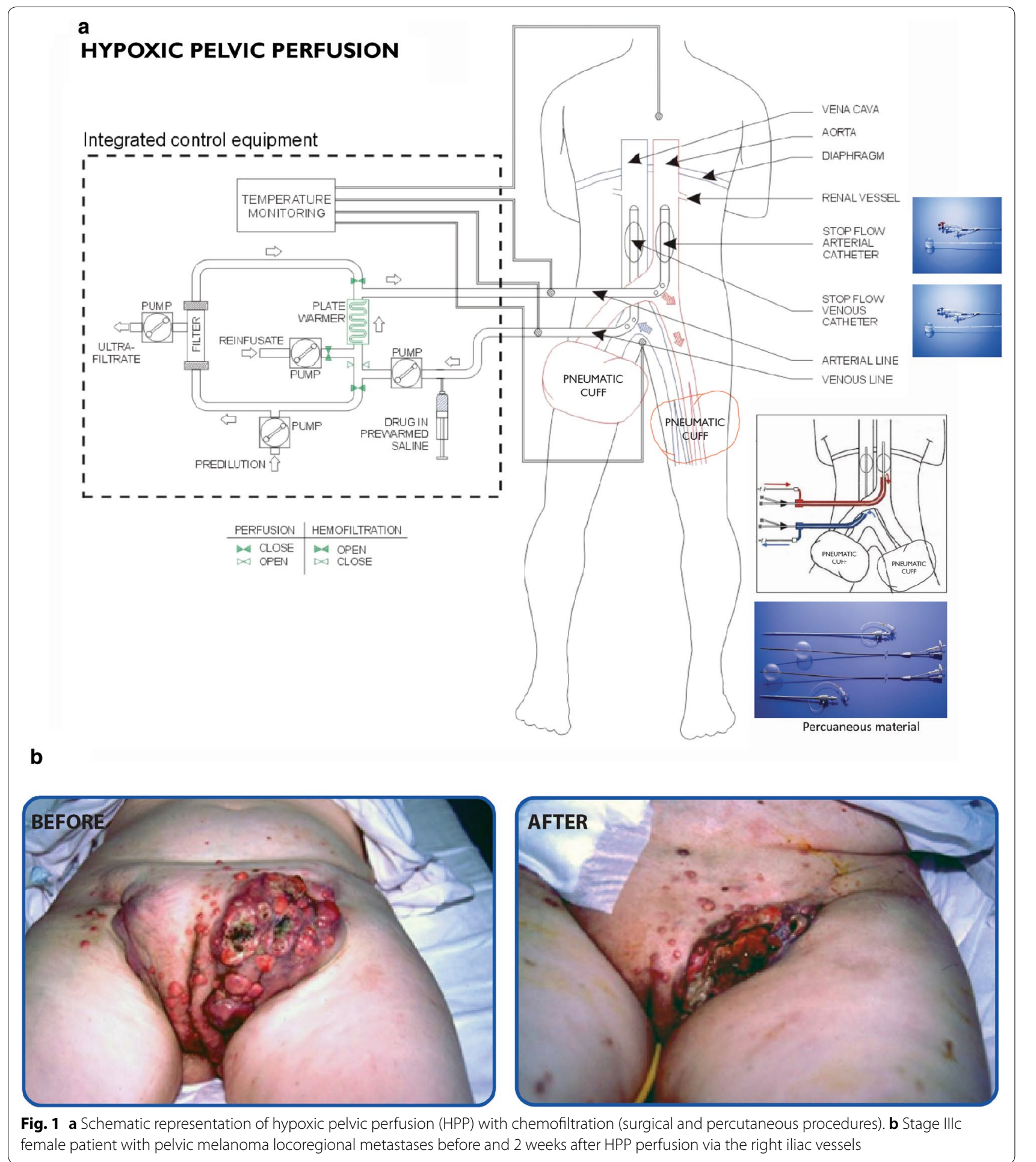

balloon catheters, introduced either percutaneously or surgically, and at thigh-level with pneumatic cuffs. Perfusion was performed under hypoxic conditions with low flow-rates (50-150 $\mathrm{ml} / \mathrm{min}$ ) and mild circuit hyperthermia in order to maintain tissue normothermia. Tumour response, according to Response Evaluation Criteria in Solid Tumors, version 1.1 [30], was assessed at $\approx 45$ days following the 1st cycle of melphalan HPP. Responses for deep masses were evaluated by Computerized Tomography (CT), Magnetic Resonance Imaging (MRI) and 
Position-emission Tomography (PET) and superficial lesions were monitored by physical inspection with photographic comparison. Statistical analysis is descriptive due to the small sample size. Purified CTC numbers, progression-free survival (PFS) and OS times are presented as medians and interquartile range. The relationship between CTC melphalan chemosensitivity and disease response to melphalan HPP are presented, without confidence intervals, as percentages of sensitivity, specificity, positive predictive value (PPV), negative predictive value (NPV) and accuracy. All computations were performed using STATA statistical software.

More than 5 viable metastatic melanoma cells $/ \mathrm{ml}$ were isolated in liquid biopsies of all 7 patients (Table 1B), median number $9.6 / \mathrm{ml}$ (interquartile range $8.4-9.8$ ). The chemosensitivity of CTCs are presented in Table 1B. As concerning melphalan, the drug used for HPP, a chemosensitivity cut-off value $>60 \%$ cell-death was observed in CTCs from 3 patients. RECIST 1.1 responses to melphalan HPP for the 7 patients are presented in Table 1A. A partial response (PR) occurred in only 1 patient (Fig. 1b). Positive (complete or partial) and negative (stable disease or progression) RECIST 1.1 responses, following melphalan HPP, associated with CTC melphalan chemosensitivity $>60 \%$ or $<60 \%$, in terms of dead/dying cells, are displayed in Table 2. A 100\% sensitivity value was observed for complete or partial RECIST 1.1 response, following melphalan HPP, associated with $>60 \%$ CTC chemosensitivity to melphalan and a $66.67 \%$ specificity value was observed for stable or progression RECIST 1.1 disease responses, following melphalan HPP, associated with $<60 \%$ CTC chemosensitivity to melphalan. A $33.33 \%$ PPV for a positive RECIST 1.1 response was associated with $>60 \%$ CTC chemosensitivity to melphalan and a 100\% NPV for a negative RECIST 1.1 response was associated with $<60 \%$ CTC chemosensitivity to melphalan. The overall capacity of CTC chemosensitivity tests to predict response to melphalan HPP (accuracy value)

Table 2 Positive (complete or partial) and negative (stable disease or progression) RECIST 1.1 responses after melphalan HPP, associated with $>60 \%$ (Positive) or $<60 \%$ (Negative) melphalan-treated CTC cell death

\begin{tabular}{llll}
\hline Chemosensitivity of CTCs & \multicolumn{2}{l}{ RECIST 1.1 response } & Total \\
\cline { 2 - 3 } & $\begin{array}{llll}\text { Positive } \\
\text { (CR + PR) }\end{array}$ & $\begin{array}{l}\text { Negative } \\
\text { (SD + PD) }\end{array}$ & \\
\hline Positive $(>60 \%)$ & 1 & 2 & 3 \\
Negative $(\leq 60 \%)$ & 0 & 4 & 4 \\
Total & 1 & 6 & 7 \\
\hline
\end{tabular}

$C R$ complete response, $P R$ partial response, $S D$ stable disease, $P D$ progressive disease of $71.43 \%$ was calculated from the ratio of positively and negatively corrected classified patients, using a melphalan chemosensitivity cut-off value of $60 \%$ and RECIST 1.1 criteria, and the total number of patients treated with melphalan HPP.

PFS (Table 1A) ranged from 2 to 4 months (median 3 months, interquartile range 3-4 months). Locoregional progression occurred in 3 patients, with locoregional plus distant site progression observed in the other 4 patients. In accordance with CTC chemosensitivity tests, each patient received chemotherapeutic agents based upon $>60 \%$ CTC chemosensitivity to that agent. Based on CTC chemosensitivity suggestions, 4 patients with locoregional plus distant metastases received systemic chemotherapy only and 3 patients with locoregional relapse received locoregional treatments (Table 1A). All patients subsequently died from disease progression, associated with OS times ranging from 7 to 47 months (median 19 months, interquartile ranges 15-21 months).

This pilot study was initiated to assess the feasibility of using purified CTCs, in terms of reproducibility, sampling, storage, transport, purification and enrichment methodologies, and the utility and suitability of subsequent CTC chemosensitivity assays in selecting therapeutic strategies and predicting response. For this purpose, we selected a homogeneous group of 7 stage IIIC melanoma patients with $B R A F$ wild-type status and locoregional metastases located exclusively to the pelvic region, all of whom were un-eligible for novel immunotherapy and were treated with melphalan HPP, in accordance with percentage $M G M T$ promoter methylation levels in tissue-specimen, as a relevant index of melphalan efficacy [14].

The numbers of metastatic melanoma CTCs from all liquid biopsies were greater that the cut-off value of $5 \mathrm{CTCs} / \mathrm{ml}$, required for chemosensitivity assays. The interval between blood sampling and qRT-PCR analysis did not exceed $80 \mathrm{~h}$, which has previously been reported to minimise gene and protein expression alterations [27]. We also report a $100 \%$ detection rate for patient CTCs, which is higher than previous reports of CTCs purified from liquid biopsies from primary cancers patients [31], which can be explained by increased numbers of CTC in high tumour burden stage IIIc metastatic melanomas. We demonstrate that the Annexin V-PE flow cytometry methodology employed for in vitro chemosensitivity assays, provided useful information concerning the CTC sensitivity to a variety of chemotherapeutic agents. In contrast to tissue validated chemosensitivity assays, CTC chemosensitivity assays do not preserve cell-tocell or cell-to-matrix interactions [32]. For this reason, a cut-off value of $>60 \%$ was chosen for CTC chemosensitivity analyses, which is higher than $>30 \%$ in tissue 
chemosensitivity tests that may better resembling tumour structure [32].

In order to evaluate the predictive potential of in vitro CTC chemosensitivity assays in prognosis and therapeutic strategy, melphalan was employed as the principle drug, with patient RECIST responses to melphalan HPP used to evaluate sensitivity, specificity, accuracy and predictive potential. This resulted in values of $100 \%$ for sensitivity, $66.67 \%$ for specificity, $33.33 \%$ for PPV, $100 \%$ for PNV and $71.43 \%$ for accuracy, which were better overall values than those of $85.7 \%$ for sensitivity, $18.2 \%$ for specificity, $40 \%$ for PPV, $66.7 \%$ for PNV and $44.44 \%$ for accuracy, reported for in vitro tissue-validated chemosensitivity assays in ovarian cancers, applying a cutoff value of $30 \%$ [32]. Our observations are also in line with predictive potential of percentage MGMT promoter methylation, which was assessed in tissue biopsies from all 7 patients and was also predictive for melphalan efficacy [14].

This pilot study supports the feasibility of a methodology for liquid biopsy sampling, storage, transport, CTC purification, transient in vitro CTC culture and subsequent use of CTCs in chemosensitivity assays. We demonstrated: (i) the numbers of CTCs purified from liquid biopsies obtained from melanoma patients with locoregional metastases, is sufficient for Annexin V-PE flow cytometry-based chemosensitivity tests; (ii) in vitro CTC chemosensitivity assays are feasible to predict RECIST 1.1 responses to locoregional melphalan chemotherapy with an accuracy value of $71.43 \%$.

\section{Limitations}

The scope of this pilot study was to provide preliminary evaluation of the feasibility of this CTC-based methodological approach, as a forerunner to a larger cohort study, depending upon results, and was not designed to evaluate treatment safety, efficacy or effectiveness [33], justifying the small sample size analysed and absence of inferential statistical analysis. Stringent recruitment parameters, such as sample homogeneity, furthermore mitigate small sample sizes, and are pre-requisites for determining feasibility [33]. We caution that the feasibility results obtained in this pilot study, quantified as percentage sensitivity, specificity, PPV, PNV and accuracy, are at most indicative and should not be extrapolated to patient inclusion and exclusion criteria not used in this study. Furthermore, the use of CTC chemosensitivity assays, such as in this report, should not yet be considered to represent a validated preliminary test for therapeutic selecting, for two fundamental reasons: (i) the current lack of accepted and standardised methodologies for CTC isolation, purification, enrichment, characterisation and use in chemosensitivity assays, and (ii) the small sample size analysed. We stress that the efficacy data presented are uncontrolled and observational.

\section{Supplementary information}

Supplementary information accompanies this paper at https://doi. org/10.1186/s13104-020-05021-5.

Additional file 1.Methodology for liquid biopsies, CTCs chemosensitivity assays, and molecular evaluation.

\section{Abbreviations}

CMs: Cutaneous melanomas; HPP: Hypoxic pelvic perfusion; OS: Overall survival; MGMT: $0^{6}$-methylguanine-DNA methyltransferase; BRAF: v-Raf murine sarcoma viral oncogene homolog B; MEK: Mitogen-activated protein kinasekinase enzymes; PD-1: Programmed cell death protein 1; CLTA-4: Cytotoxic T-lymphocyte-associated protein 4; CTCs: Circulating tumor cells; USA: United States of America; RECIST: Response evaluation criteria in solid tumors; qRTPCR: Quantitative reverse transcription polymerase chain reaction; PPV: Predictive positive value; PNV: Predictive negative value; FFPE: Formalin fixed paraffin embedded; CAST: Competitive allele specific technology; PCR: Polymerase chain reaction; MS-MLPA: Methylation-Specific Multiplex Ligation-Dependent Probe Amplification; Hha1: Calpastatin Hha1 gene; EDTA: Ethylenediaminetetraacetic acid; CD45: Protein tyrosine phosphatase, receptor type, C; CD63: Protein encoded by the CD63 gene; FBS: Fetal bovine serum; RPMI-1640: Growth medium used in cell culture; CD31: Platelet endothelial cell adhesion molecule; CT: Computerized tomography; MRI: Magnetic resonance imaging; PET: Positron-emission tomography; SD: Standard deviation; PFS: Progression free survival.

\section{Acknowledgements}

We would like to thank Aigner Karl Reinhard for his surgical teaching.

\section{Authors' contributions}

SG and MC performed surgical procedures; SG, MC, GF, and ARM participated in the design of this study; DS evaluated data; IP and PA performed analyses on liquid biopsies; FM performed statistical analyses; SG, IP, and ARM drafted this manuscript; ARF revised and validated this manuscript. All authors read and approved the final manuscript.

\section{Funding}

No funding was received.

\section{Availability of data and materials}

All data generated or analysed during this study are included in this published article and are available from the corresponding author on reasonable request.

\section{Ethics approval and consent to participate}

This project has been performed in accordance with the Declaration of Helsinki and has been approved by the ethics committee of ASL n.1, Abruzzo, Italy (Chairperson: G. Piccioli; protocol number 10/CE/2018; date of approval: 19 July, 2018 (n. 1419), L'Aquila, Italy). Written informed consent was obtained from each of the 7 patients.

\section{Consent to publish}

The manuscript does not contain any individual person data, so consent for publication is not applicable.

\section{Competing interests}

The authors declare that they have no competing interests.

\section{Author details}

${ }^{1}$ Department of Applied Clinical Sciences and Biotechnology, University of L'Aquila, 67100 L'Aquila, Italy. ${ }^{2}$ Department of Oncology and Hematology, Ospedali Riuniti Marche Nord, 61121 Pesaro, Italy. ${ }^{3}$ Research Genetic Cancer 
Centre International GmbH, Zug, Switzerland. ${ }^{4}$ Research Genetic Cancer Centre S.A, Florina, Greece.

Received: 18 February 2020 Accepted: 13 March 2020

Published online: 24 March 2020

\section{References}

1. Turley RS, Raymond AK, Tyler DS. Regional treatment strategies for intransit melanoma metastasis. Surg Oncol Clin N Am. 2011;20:79-103.

2. Trout AT, Rabinowitz RS, Platt JF, Elsayes KM. Melanoma metastases in the abdomen and pelvis: frequency and patterns of spread. World J Radiol. 2013;5:25-32

3. Guadagni S, Fiorentini G, Clementi M, et al. Melphalan hypoxic perfusion with hemofiltration for melanoma locoregional metastases in the pelvis. J Surg Res. 2017;215:114-24.

4. Dong XD, Tyler D, Johnson JL, DeMatos P, Seigler HF. Analysis of prognosis and disease progression after local recurrence of melanoma. Cancer. 2000:88:1063-71.

5. Read RL, Thompson JF. Managing in-transit melanoma metastases in the new era of effective systemic therapies for melanoma. Expert Rev Clin Pharmacol. 2019. https://doi.org/10.1080/17512433.2019.168912.

6. Thompson JF, Agarwala SS, Smithers BM, Ross MI, Scoggins CR, Coventry $\mathrm{BJ}$, et al. Phase 2 study of intralesional PV-10 in refractory metastatic melanoma. Ann Surg Oncol. 2015;22:2135-42.

7. Chesney J, Puzanov I, Collichio F, Singh P, Milhem MM, Glaspy J, et al. Randomized, open-label phase II study evaluating the efficacy and safety of talimogene laherparepvec in combination with ipilimumab versus ipilimumab alone in patients with advanced, unresectable melanoma. J Clin Oncol. 2017:36:1658-67.

8. Campana L, Valpione S, Mocellin S, Sundararajan R, Granziera E, Sartore L, et al. Electrochemotherapy for disseminated superficial metastases from malignant melanoma. Br J Surg. 2012;99:821-30.

9. Caracò C, Marone U, Simeone E, Grimaldi AM, Botti G, Del Giudice M, et al. Electrochemotherapy in melanoma patients: a single institution experience. Mel Manag. 2015;2:127-32.

10. Garbe C, Amaral T, Peris K, Hauschild A, Arenberger P, Bastholt L, On behalf of the European Dermatology Forum (EDF), The European Association of Dermato-Oncology (EADO), The European Organization for Research and Treatment of Cancer (EORTC), et al. European consensus-based interdisciplinary guideline for melanoma. Part 2: treatment—update 2019. Eur J Cancer. 2019. https://doi.org/10.1016/j. ejca.2019.11.015.

11. Hong A, Fogarty G. Role of radiation therapy in cutaneous melanoma. Cancer J. 2012:18:203-7.

12. Fiorentini G, Cantore M, Montagnani F, Mambrini A, D'Alessandro M, Guadagni S. The role of hypoxia and hyperthermia in chemotherapy. In: Aigner KR, Stephens FO, editors. Induction chemotherapy. Systemic and locoregional. 2nd ed. Berlin: Springer-Verlag; 2016. p. 61-71.

13. Guadagni S, Palumbo G, Fiorentini G, Clementi M, Marsili L. Surgical versus percutaneous isolated pelvic perfusion (IPP) for advanced melanoma: comparison in terms of melphalan pharmacokinetic pelvic bio-availability. BMC Res Notes. 2017;10:411.

14. Guadagni S, Fiorentini G, Clementi M, et al. MGMT methylation correlates with melphalan pelvic perfusion survival in stage III melanoma patients: a pilot study. Melanoma Res. 2017;27:439-47.

15. Guadagni S, Fiorentini G, Clementi M, et al. Does locoregional chemotherapy still matter in the treatment of advanced pelvic melanoma? Int J Mol Sci. 2017;18:2382. https://doi.org/10.3390/ijms18112382.

16. Tie EN, Lai-Kwon JE, Gyorki DE. Systemic therapies for unresectable locoregional melanoma: a significant area of need. Melanoma Manag. 2019. https://doi.org/10.2217/mmt-2019-0010
17. Khushalani NI, Sondak VK. Are we there yet? Prolonged MAPK inhibition in BRAF V600-mutant melanoma. Lancet Oncol. 2016;17:1178-9.

18. Chan MM, Haydu LE, Menzies AM, et al. The nature and management of metastatic melanoma after progression on BRAF inhibitors: effects of extended BRAF inhibition. Cancer. 2014:120:3142-53.

19. Topollian SL, Sznol M, McDermott DF, et al. Survival, durable tumor remission, and long-term safety in patients with advanced melanoma receiving nivolumab. J Clin Oncol. 2014;32:1020-30.

20. Ribas A, Hamid O, Daud A, et al. Association of pembrolizumab with tumor response and survival among patients with advanced melanoma. JAMA. 2016;315:1600-9.

21. Daud A, Nandoskar P. Pembrolizumab for melanoma-safety profile and future trends. Expert Opin Drug Saf. 2016;15:727-9.

22. Johnson DB, Sullivan RJ, Menzies AM. Immune checkpoint inhibitors in challenging populations. Cancer. 2017;123:1904-11.

23. Karachaliou N, Mayo-de-Las-Casas C, Molina-Vila MA, Rosell R. Real-time liquid biopsies become a reality in cancer treatment. Ann Transl Med. 2015;3:36.

24. Guadagni S, Fiorentini G, De Simone M, et al. Precision oncotherapy based on liquid biopsies in multidisciplinary treatment of unresectable recurrent rectal cancer: a retrospective cohort study. J Cancer Res Clin Oncol. 2020;146:205-19.

25. Lim SB, Lim CT, Lim W-T. Single-cell analysis of circulating tumor cells: why heterogeneity matters. Cancers. 2019;11:1595. https://doi. org/10.3390/cancers11101595.

26. Beasley A, Isaacs T, Khattak MA. Clinical application of circulating tumor cells and circulating tumor DNA in uveal melanoma. JCO Precis Oncol. 2018. https://doi.org/10.1200/PO.17.00279.

27. Apostolou P, Ntanovasilis DA, Papasotiriou I. Evaluation of a simple method for storage of blood samples that enables isolation of circulating tumor cells $96 \mathrm{~h}$ after sample collection. J Biol Res Thessalon. 2017:24:11.

28. Apostolou P, Toloudi M, Chatziioannou M, et al. Anvirzel ${ }^{\mathrm{TM}}$ in combination with cisplatin in breast, colon, lung, prostate, melanoma and pancreatic cancer cell lines. BMC Pharmacol Toxicol. 2013;14:18.

29. Guadagni S, Kanavos E, Schietroma M, Fiorentini G, Amicucci G. Selected hypoxic stop-flow perfusions: indication and limits. Tumori. 2006;92:402-6.

30. Eisenhauer EA, Therasse P, Bogaerts J. New response valuation criteria in solid tumors: Revised RECIST guideline (version 1.1). Eur J Cancer. 2009:45:228-47.

31. Asante D, Calaprea L, Ziman L, Meniawy TM, Gray ES. Liquid biopsy in ovarian cancer using circulating tumor DNA and cells: ready for prime time? Cancer Lett. 2020;468:59-71.

32. Kim J-H, Yoon YS, Kim JC, Kim Y-M. Assessment of the applicability of Integrative tumor response assays in advanced epithelial ovarian cancer. Anticancer Res. 2019;39:313-8.

33. Leon AC, Davis LL, Kraemer HC. The role and interpretation of pilot studies in clinical research. J Psychiatr Res. 2011;45:626-9.

\section{Publisher's Note}

Springer Nature remains neutral with regard to jurisdictional claims in published maps and institutional affiliations.

Ready to submit your research? Choose BMC and benefit from:

- fast, convenient online submission

- thorough peer review by experienced researchers in your field

- rapid publication on acceptance

- support for research data, including large and complex data types

- gold Open Access which fosters wider collaboration and increased citations

- maximum visibility for your research: over $100 \mathrm{M}$ website views per year

At BMC, research is always in progress.

Learn more biomedcentral.com/submissions 